\title{
Comprehensive analyses of the immunoglobulin proteome for the classification of glomerular diseases
}

Jianhua $\mathrm{Liu}^{\dagger, \S}$, Yang $\mathrm{Li}^{\ddagger}{ }^{\ddagger}$, Jiayu Dai ${ }^{\ddagger}$, Baoxu $\mathrm{Lin}^{\dagger}$, Chunying Xiao ${ }^{\dagger}$, Xinpeng Zhang ${ }^{\dagger}$, Lin Luo ${ }^{\dagger}$, Tingting $\mathrm{Wang}^{\dagger}$, Xiaoying $\mathrm{Li}^{\dagger}$, Yao $\mathrm{Yu}^{\dagger}$, Shixiao $\mathrm{Chen}^{\dagger}$, Lina $\mathrm{Wu}^{\dagger}$, Yong Liu $^{\dagger}$, Xiaobo $\mathrm{Yu}^{\ddagger}{ }^{\ddagger}$, Xiaosong Qin ${ }^{\dagger, *}$.

${ }^{\S}$ Contributed equally to this article.

* Senior authors contributed equally to this article.

$\dagger$ Department of Laboratory Medicine, Shengjing Hospital of China Medical University, Shenyang 110004, China

${ }^{\ddagger}$ State Key Laboratory of Proteomics, Beijing Proteome Research Center, National Center for Protein Sciences-Beijing (PHENIX Center), Beijing Institute of Lifeomics, Beijing 102206, China.

Correspondence and requests for materials should be addressed to X.Q. (qinxs@sj-hospital.org ; Tel: 86 (24)96615-72130; Fax: 86 (24)96615-72116) or X.Y. (yuxiaobo@mail.ncpsb.org; Tel: 86 (10)61777093; Fax: 86 (10)61777050).

Supplementary Tables: 2

Supplemental Figures: 16

\section{Supplemental Tables}

Table S1. Acronyms and full description of clinical data variables used in this study.

Table S2. Demographic and clinical information of DN patients. 


\section{Supplemental Figures}

Figure S1

Figure S2

Figure S3

Figure S4

Figure S5

Figure S6

Figure S7

Figure S8

Figure S9

Non-hierarchical clustering analyses of the correlations between
any two variables of the immunoglobulin proteome and clinical

Non-hierarchical clustering analyses of the correlations between
any two variables of the immunoglobulin proteome and clinical data in IMN patients.

Figure S10

The standard curves obtained for quantifying immunoglobulins using our high throughput serum microarray.

Inter-assay correlation of serological $\operatorname{IgA}$ detection using our serum microarray.

Influence of printing location on the reproducible detection of immunoglobulin proteins.

Specificity of eight anti-human immunoglobin antibodies.

Network analysis of the immunoglobulin proteome correlations in different glomerular diseases.

Correlation analysis of the immunoglobulin proteome in different glomerular diseases.

Non-hierarchical clustering analyses of the correlations between any two variables of the immunoglobulin proteome and clinical data in healthy controls

Non-hierarchical clustering analyses of the correlations between any two variables of the immunoglobulin proteome and clinical data in IgAN patients.

Non-hierarchical clustering analyses of the correlations between 
any two variables of the immunoglobulin proteome and clinical data in MCN patients.

Figure S11 Non-hierarchical clustering analyses of the correlations between any two variables of the immunoglobulin proteome and clinical data in FSGS patients.

Figure S12 Non-hierarchical clustering analyses of the correlations between any two variables of the immunoglobulin proteome and clinical data in DN patients.

Figure S13 Non-hierarchical clustering analyses of the correlations between any two variables of the immunoglobulin proteome and clinical data in LN patients.

Figure S14 Non-hierarchical clustering analyses of the correlations between any two variables of the immunoglobulin proteome and clinical data in HSPN patients.

Figure S15 Non-hierarchical clustering analyses of the correlations between any two variables of the immunoglobulin proteome and clinical data in ANCA-AG patients.

Figure S16 Validation correlation between the expression of serum $\operatorname{IgG}$ and $\mathrm{HbA1c}$ in DN patients 
Table S1. Acronyms and full description of clinical data variables used in this study.

\begin{tabular}{|c|c|}
\hline Abbreviation & Full name \\
\hline $\mathrm{Cr}$ & creatinine \\
\hline Ure & urea \\
\hline Cys-C & cystatin- $\mathrm{C}$ \\
\hline eGFR & estimated glomerular filtration rate \\
\hline 24hUP & 24 hour urine protein \\
\hline $\mathbf{T P}$ & total protein \\
\hline ALB & albumin \\
\hline ALP & alkaline phosphatase \\
\hline GGT & gamma-glutamyl transferase \\
\hline ALT & alanine aminotransfease \\
\hline AST & aspartate aminotransferase \\
\hline TBIL & total bilirubin \\
\hline DBIL & direct bilirubin \\
\hline TBA & total bile acid \\
\hline LDH & lactate dehydrogenase \\
\hline TC & total cholesterol \\
\hline TG & triglyceride \\
\hline LDL-C & low density lipoprotein cholesterol \\
\hline HDL-C & High-density lipoprotein cholesterol \\
\hline Glu & glucose \\
\hline PT & prothrombin time \\
\hline INR & international normalized ratio \\
\hline APTT & activated partial thromboplastin time \\
\hline TT & thrombin time \\
\hline DD & D-dimer \\
\hline FDPs & fibrinogen/fibrin degradation products \\
\hline uRBC & urine red blood cell \\
\hline WBC & white blood cell \\
\hline NEU & neutrophil \\
\hline LYM & lymphocyte \\
\hline RBC & red blood cell \\
\hline PLT & platelet \\
\hline HGB & Hemoglobin \\
\hline CRP & C-reactive protein \\
\hline $\mathrm{C3}$ & complement 3 \\
\hline
\end{tabular}



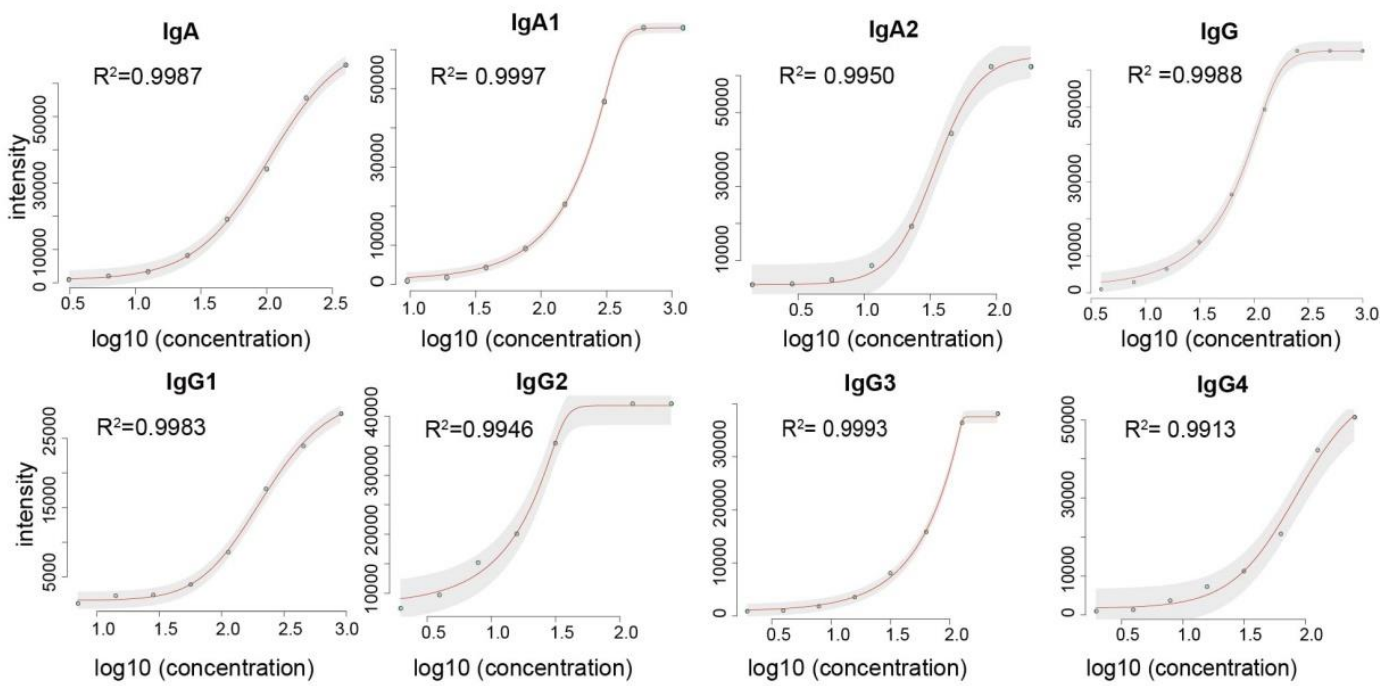

Figure S1. The standard curves obtained for quantifying immunoglobulins using our high throughput serum microarray. 


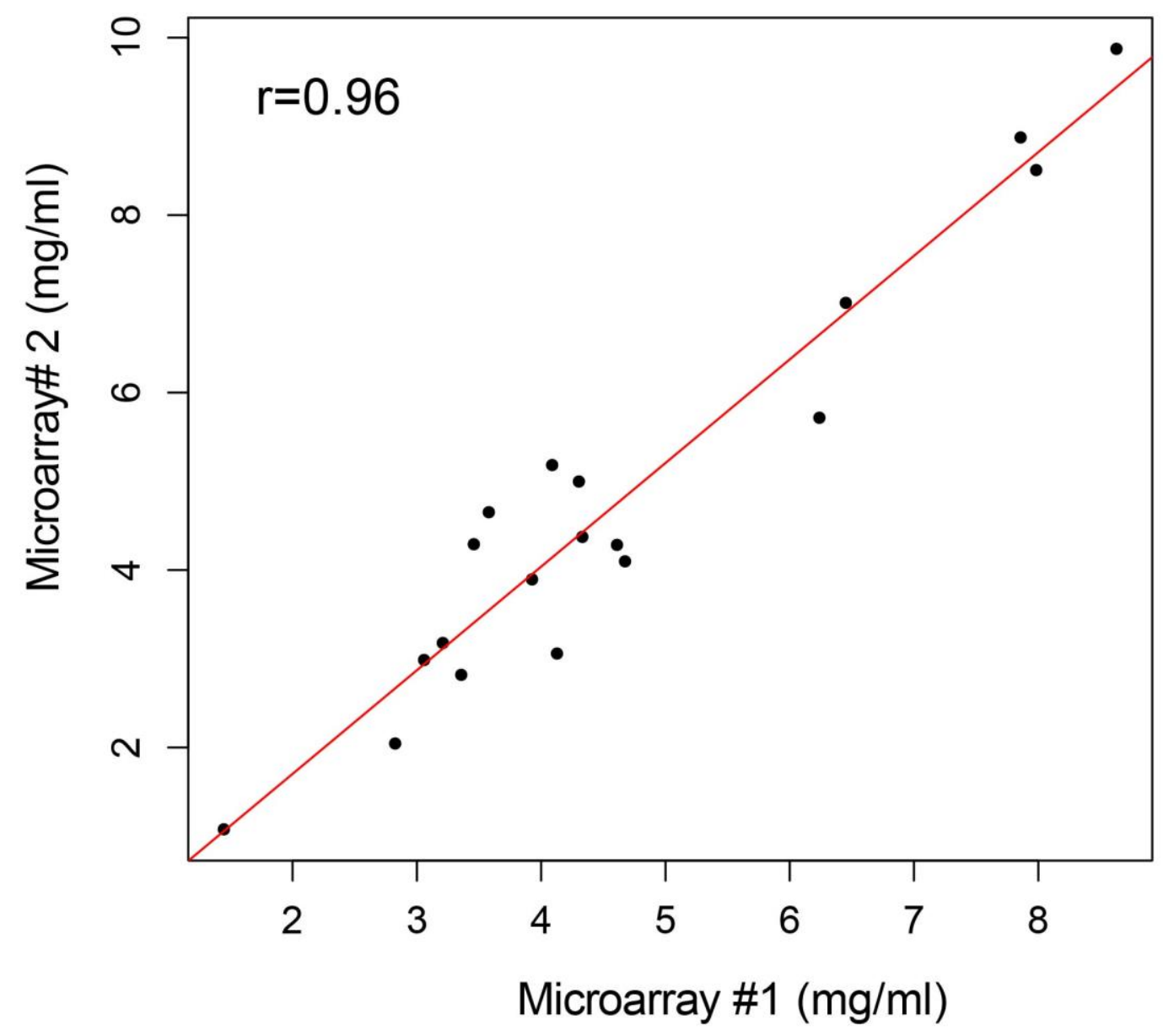

Figure S2. Inter-assay correlation of serological IgA detection using serum microarray. 

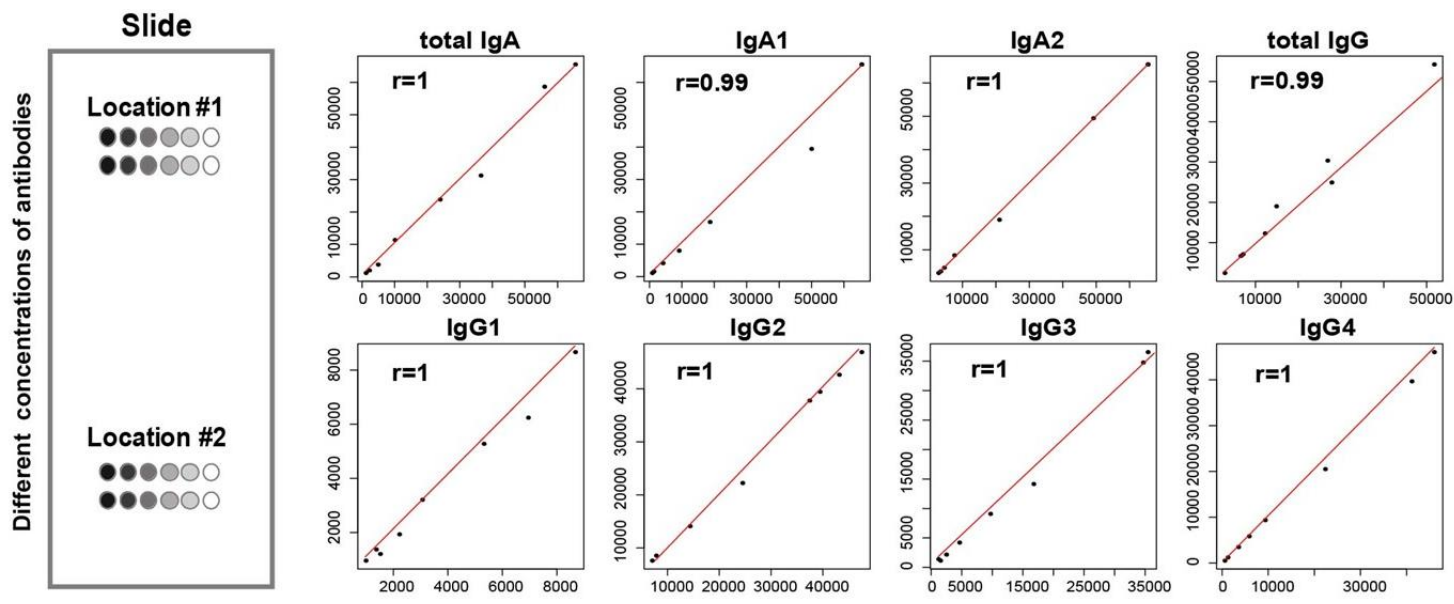

Figure S3. Influence of printing location on the reproducible detection of immunoglobulin proteins. 

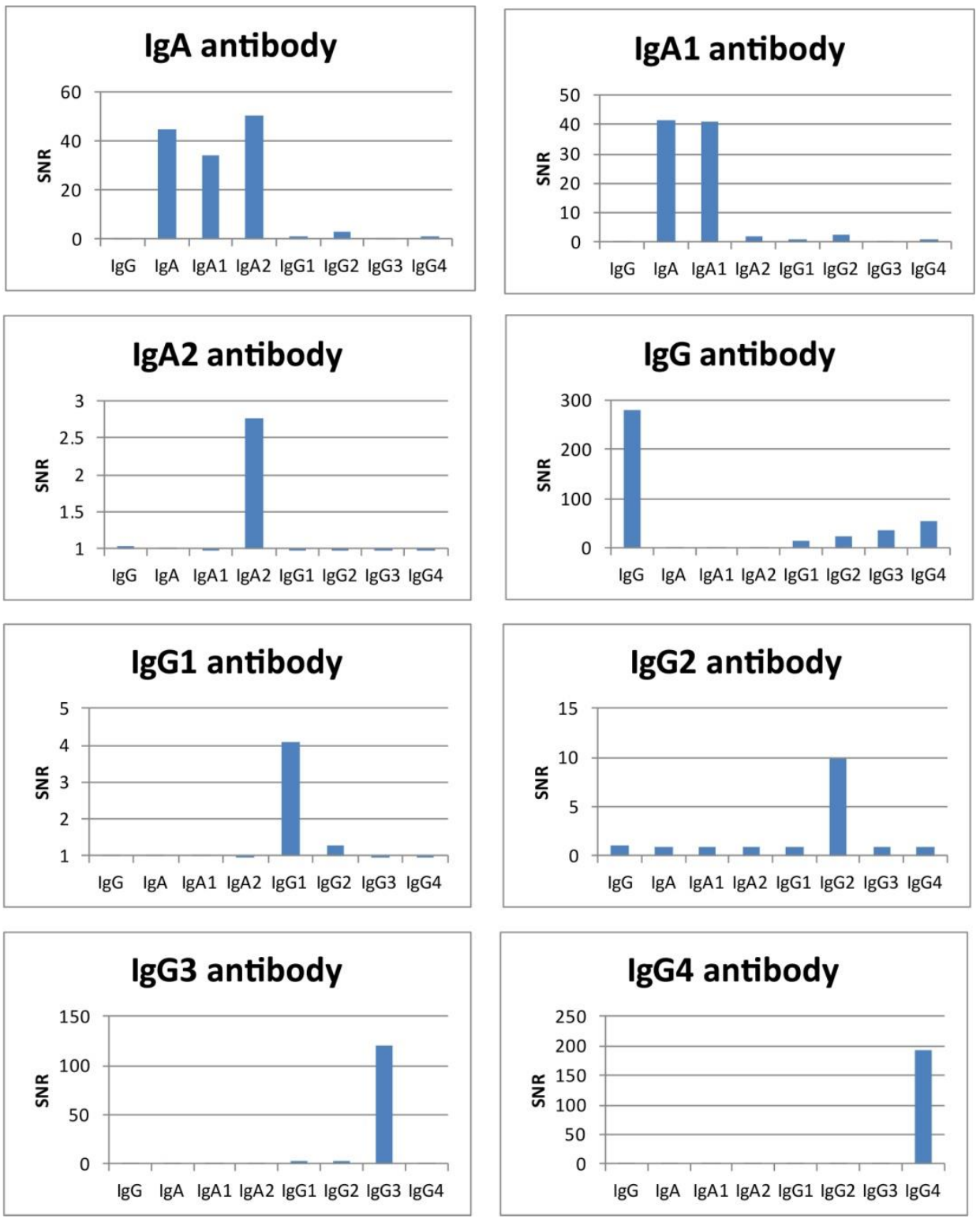

Figure S4 Specificity of eight anti-human immunoglobin antibodies. SNR: signal to noise ratio. 

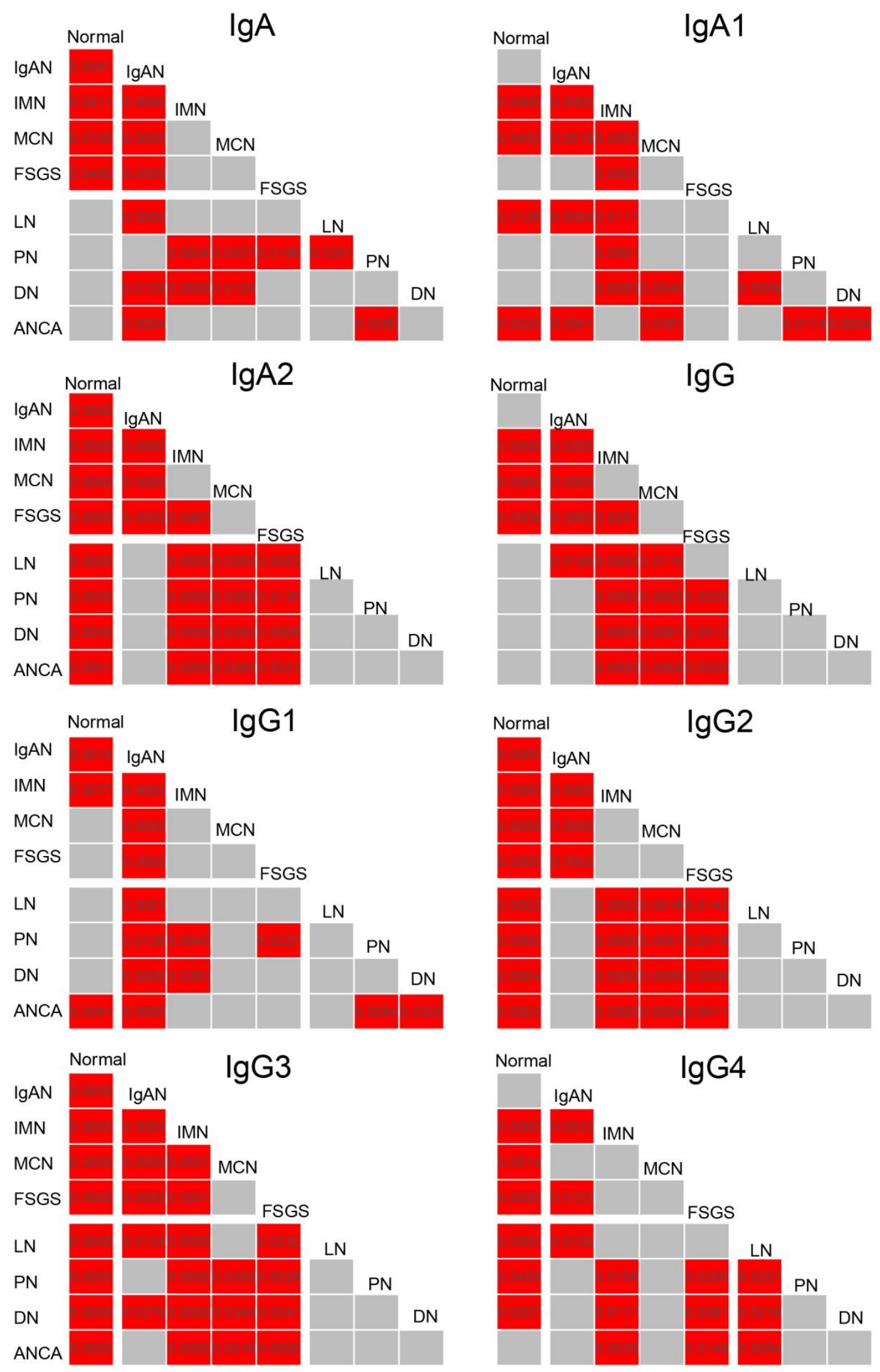

Figure S5. Network analysis of the immunoglobulin proteome correlations in different glomerular diseases. The Pearson's or Spearman's correlation coefficient 
was calculated between two variables according to their normality as described in the Methods section.

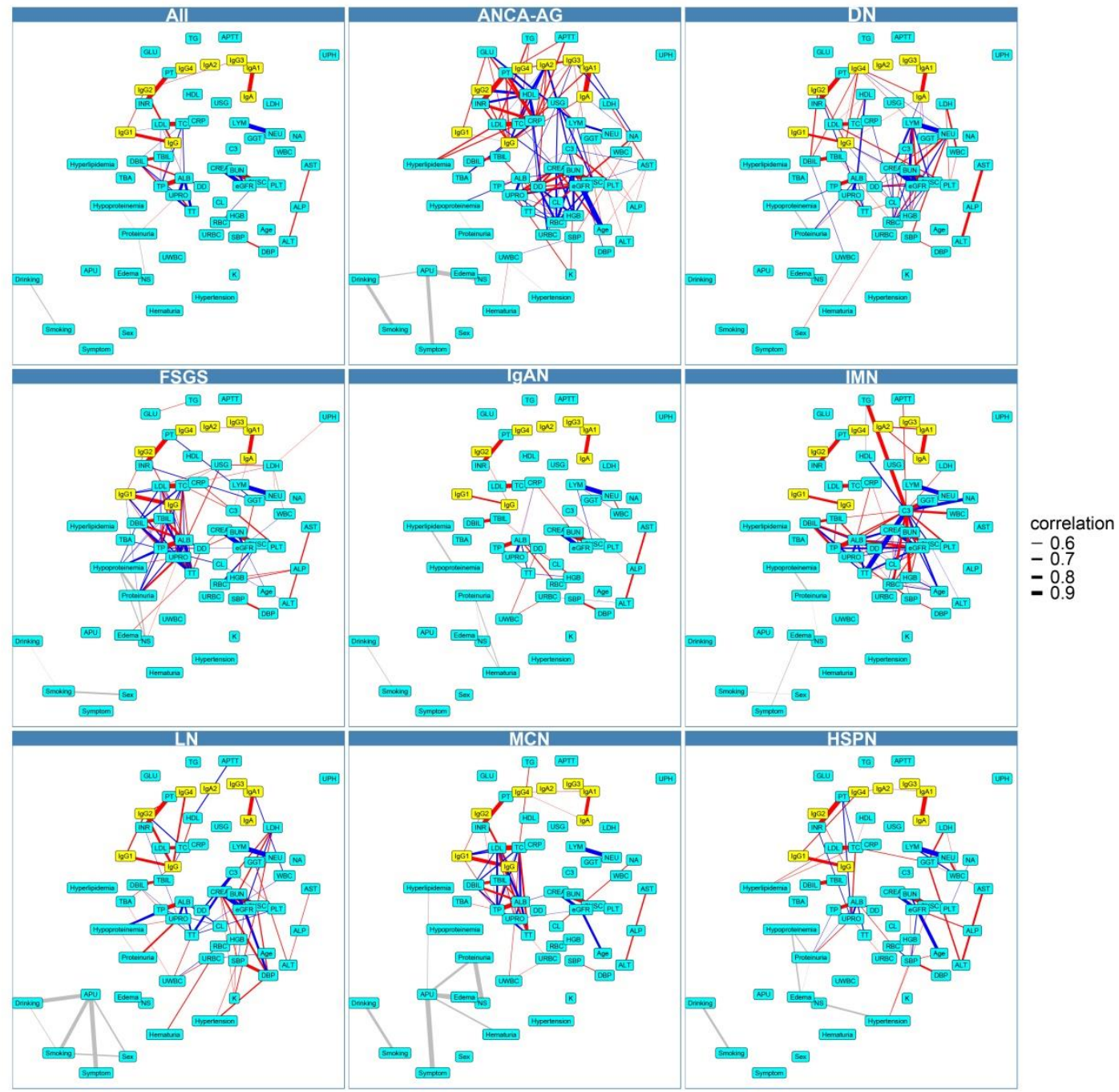

Figure S6. Correlation analysis of the immunoglobulin proteome in different glomerular diseases. 


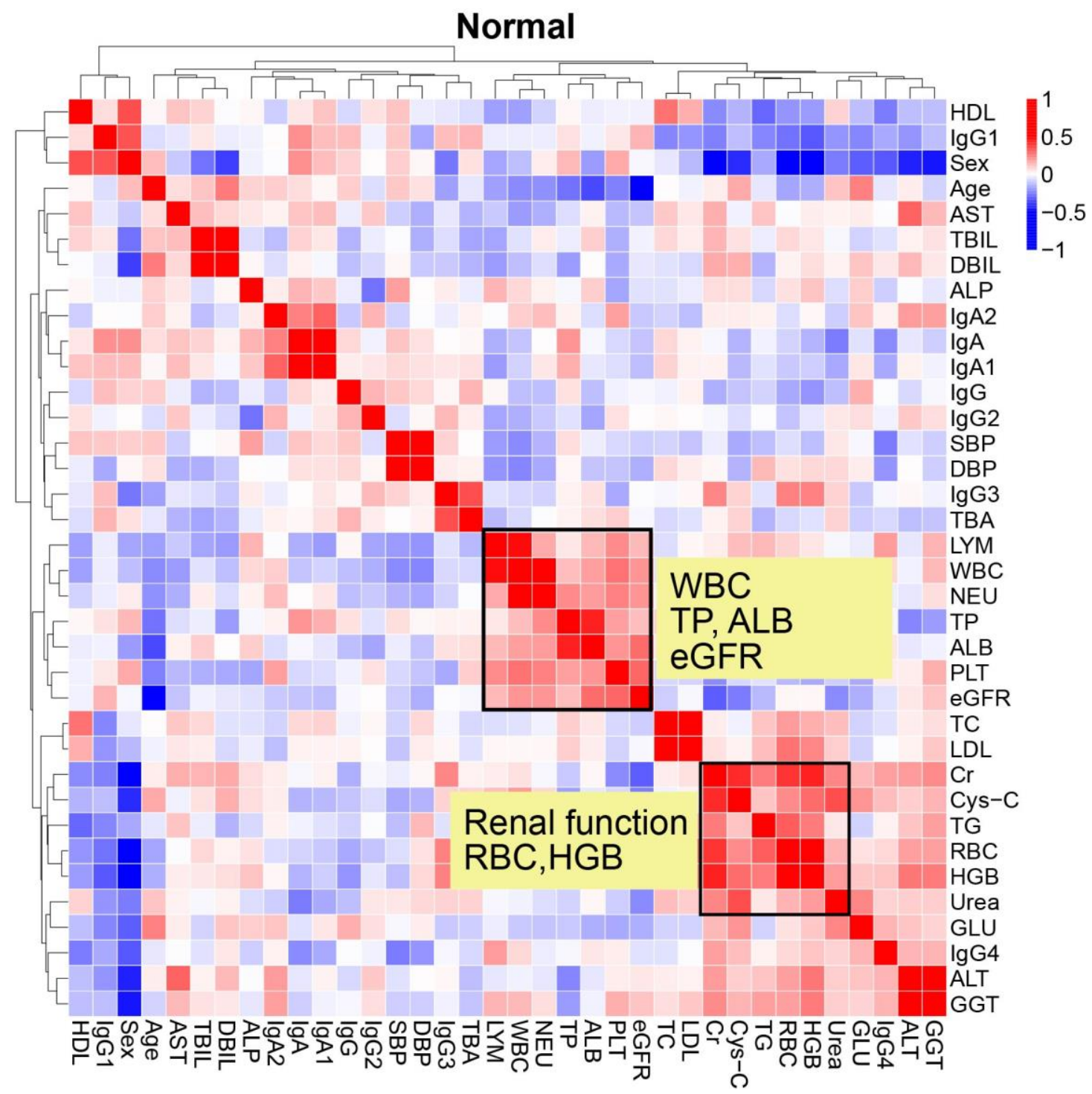

Figure S7. Non-hierarchical clustering analyses of the correlations between any two variables of the immunoglobulin proteome and clinical data in healthy controls. The rainbow color from blue to red corresponds to the correlation between two variables from low to high, respectively. 


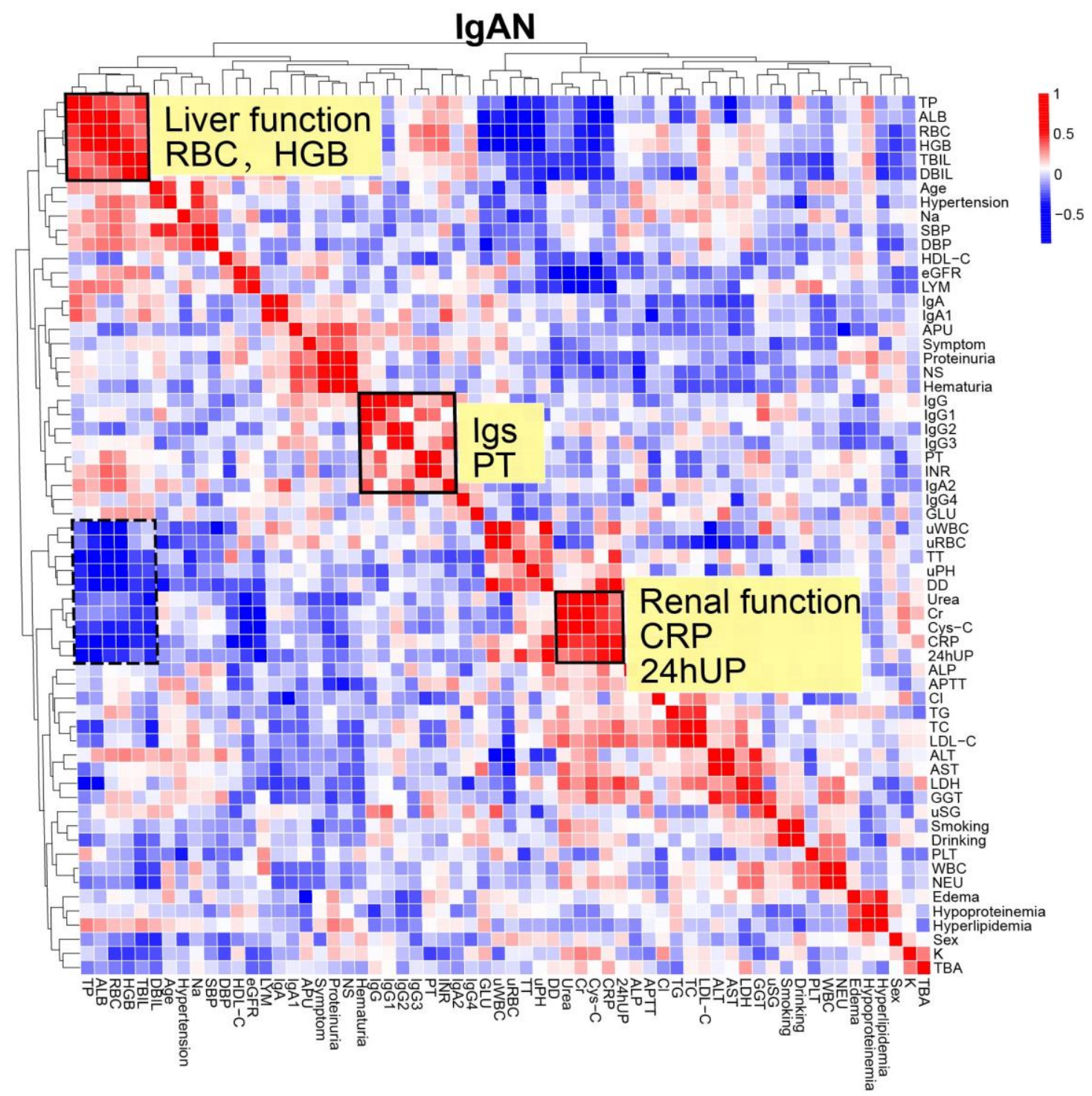

Figure S8. Non-hierarchical clustering analyses of the correlations between any two variables of the immunoglobulin proteome and clinical data in IgAN patients. The rainbow color from blue to red corresponds to the correlation between two variables from low to high, respectively. 


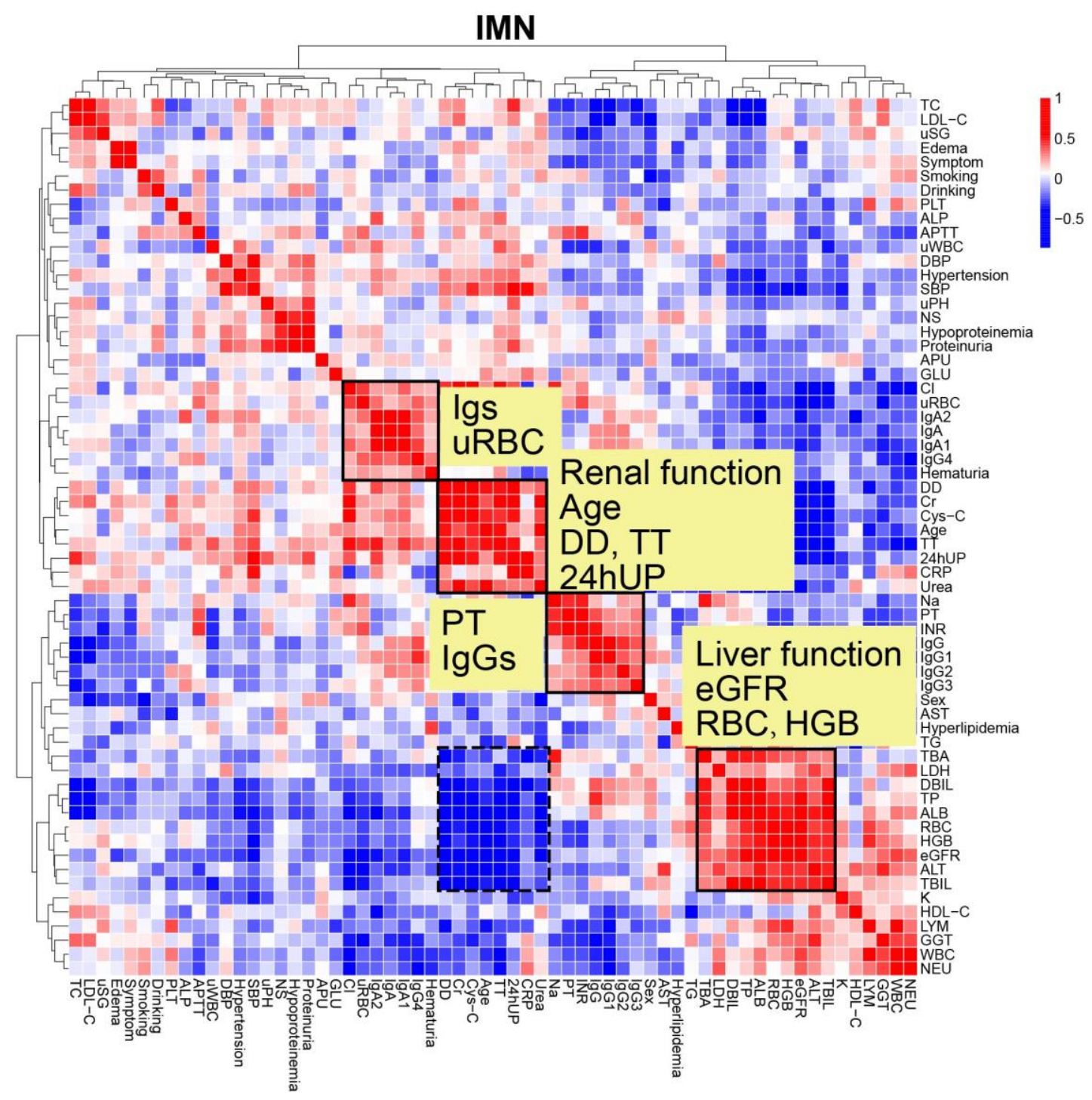

Figure S9. Non-hierarchical clustering analyses of the correlations between any two variables of the immunoglobulin proteome and clinical data in IMN patients. The rainbow color from blue to red corresponds to the correlation between two variables from low to high, respectively. 


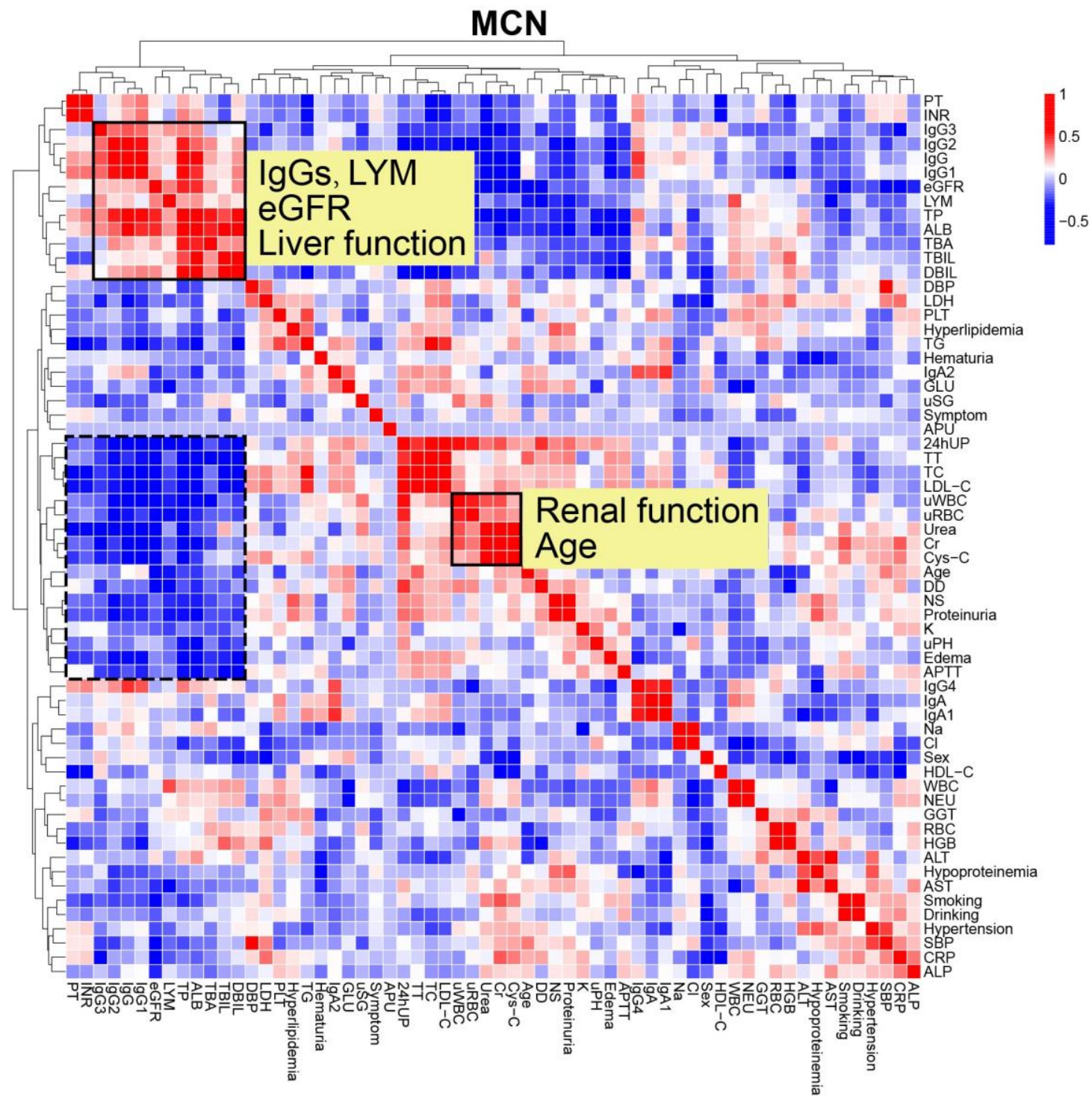

Figure S10. Non-hierarchical clustering analyses of the correlations between any two variables of the immunoglobulin proteome and clinical data in MCN patients. The rainbow color from blue to red corresponds to the correlation between two variables from low to high, respectively. 


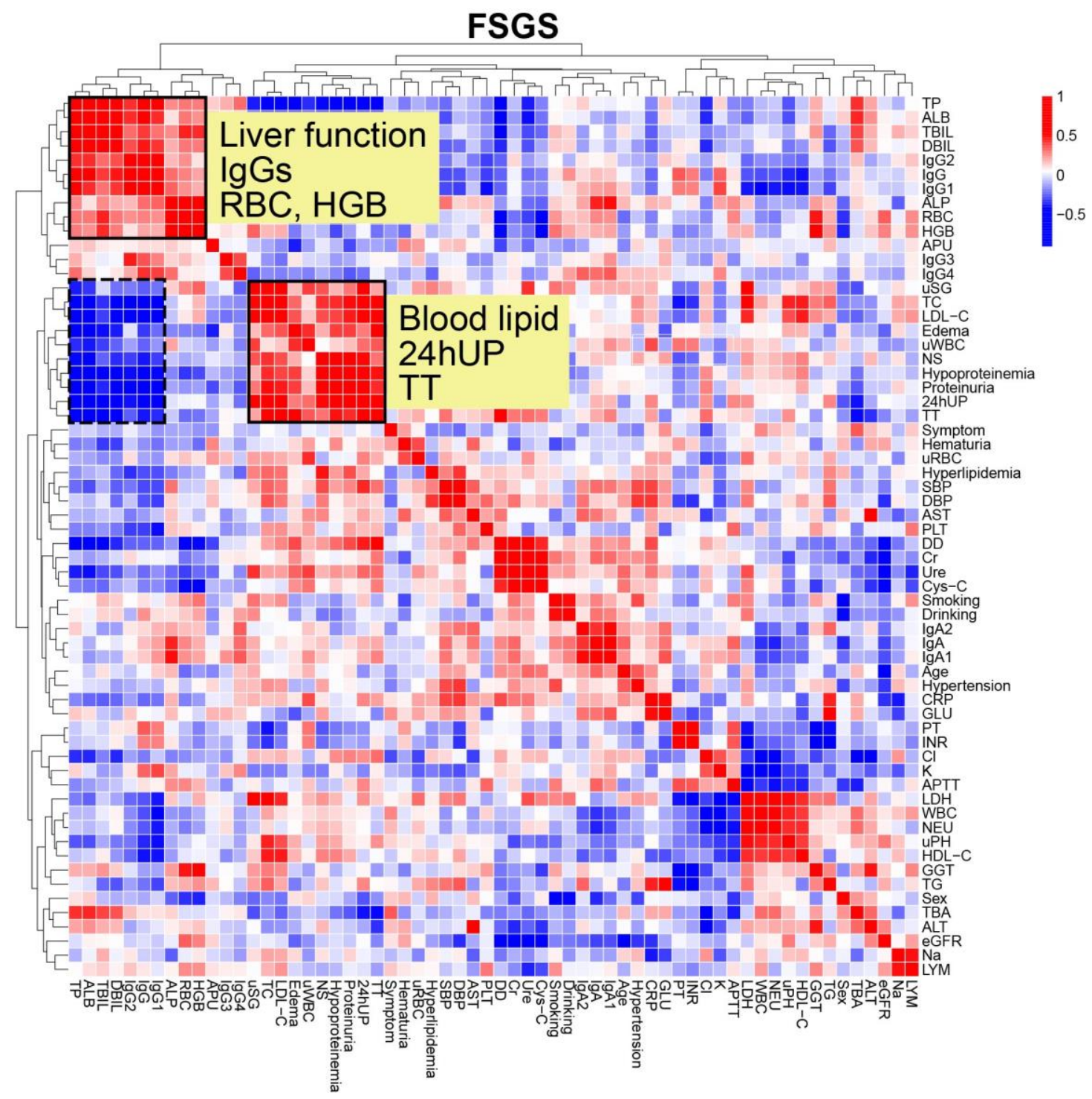

Figure S11. Non-hierarchical clustering analyses of the correlations between any two variables of the immunoglobulin proteome and clinical data in FSGS patients. The rainbow color from blue to red corresponds to the correlation between two variables from low to high, respectively. 


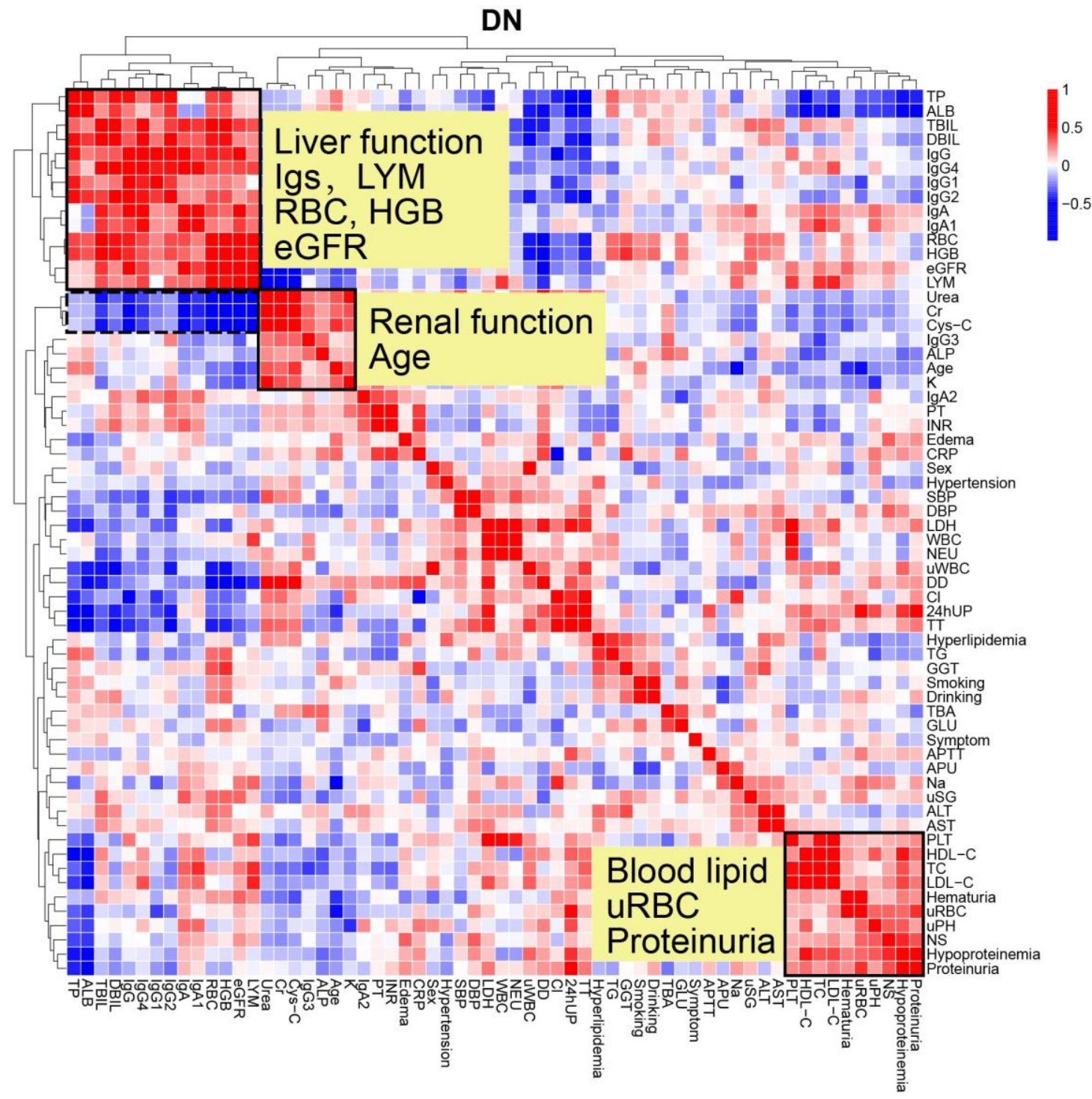

Figure S12. Non-hierarchical clustering analyses of the correlations between any two variables of the immunoglobulin proteome and clinical data in DN patients. The rainbow color from blue to red corresponds to the correlation between two variables from low to high, respectively. 


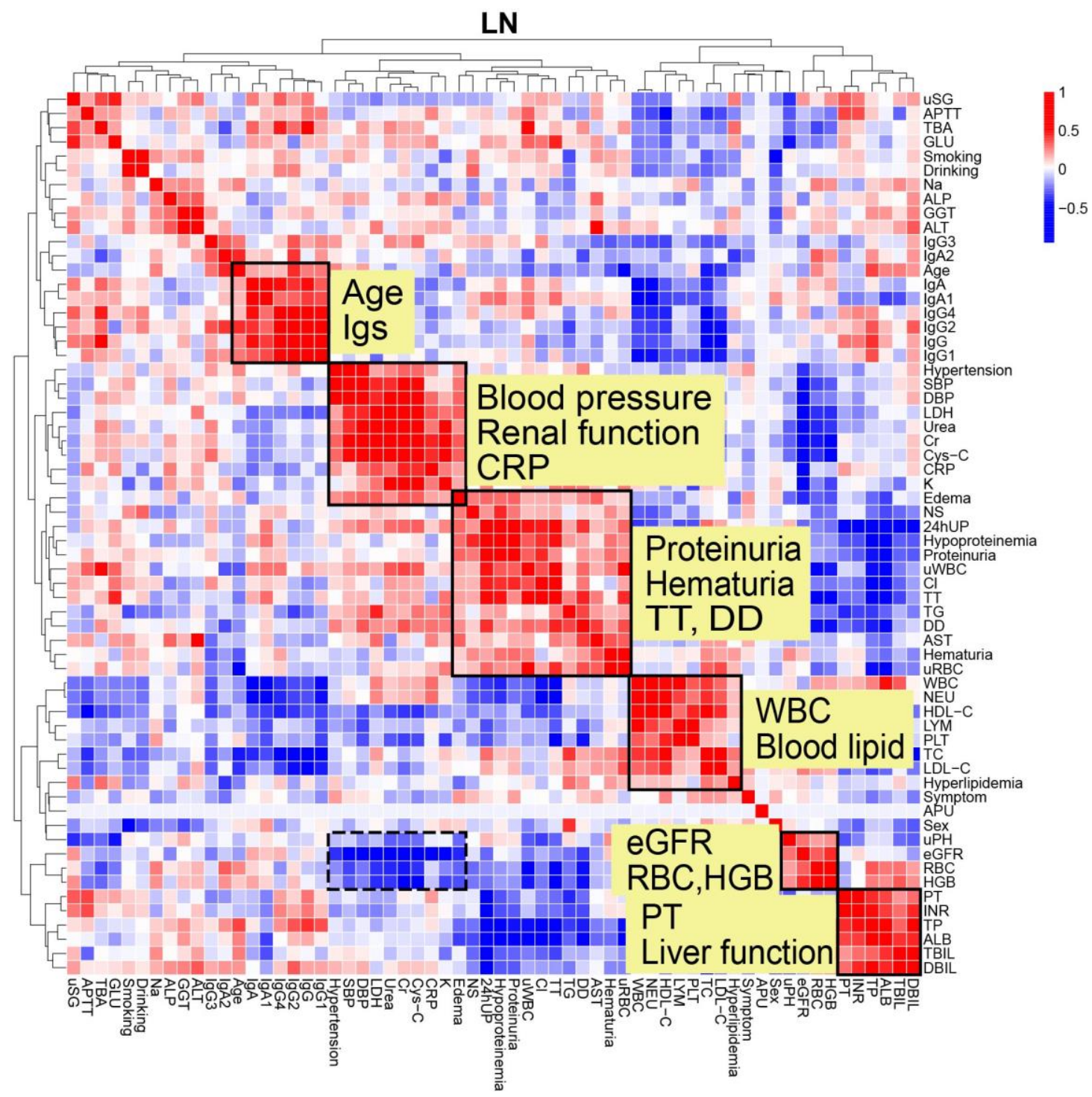

Figure 13. Non-hierarchical clustering analyses of the correlations between any two variables of the immunoglobulin proteome and clinical data in LN patients. The rainbow color from blue to red corresponds to the correlation between two variables from low to high, respectively. 


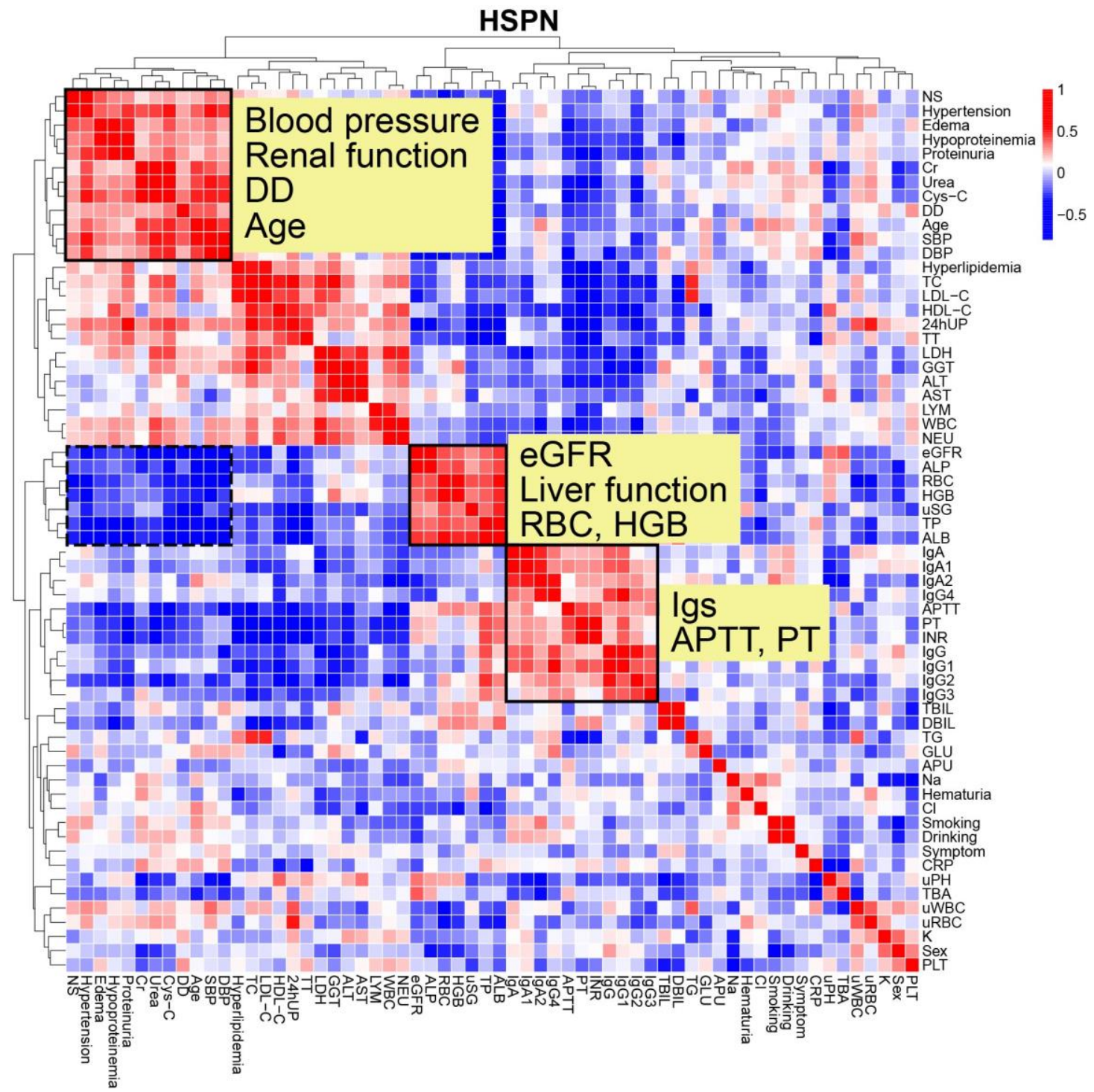

Figure S14. Non-hierarchical clustering analyses of the correlations between any two variables of the immunoglobulin proteome and clinical data in HSPN patients. The rainbow color from blue to red corresponds to the correlation between two variables from low to high, respectively. 


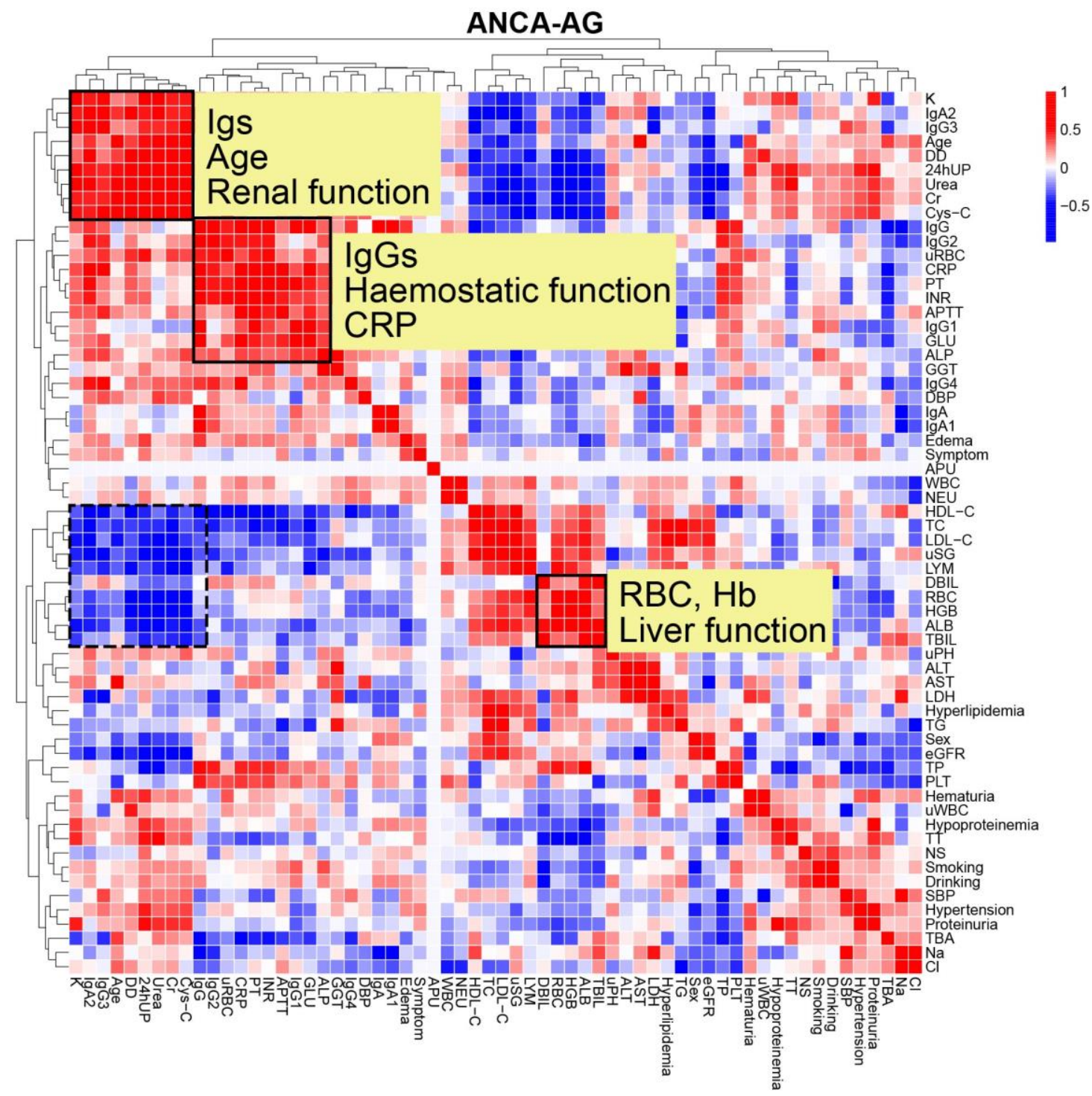

Figure S15. Non-hierarchical clustering analyses of the correlations between any two variables of the immunoglobulin proteome and clinical data in ANCA-AG patients. The rainbow color from blue to red corresponds to the correlation between two variables from low to high, respectively. 


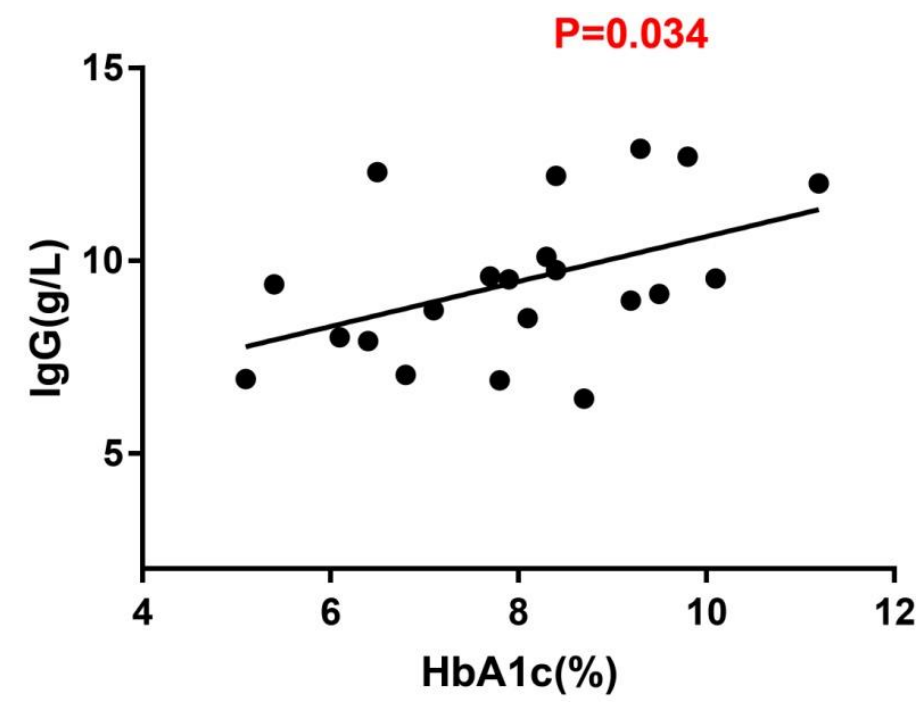

Figure S16 Validation correlation between the expression of serum IgG and HbA1c in DN patients.

Table S2. Demographic and clinical information of DN patients

\begin{tabular}{ll}
\hline Parameters & DN patients \\
\hline Gender(male/female) & $14 / 7$ \\
Age $($ year $)$ & $52.00 \pm 8.73$ \\
FBG $(\mathrm{mmol} / \mathrm{L})$ & $6.45(4.68,10.66)$ \\
$\mathrm{HbA} 1 \mathrm{c}(\%)$ & $8.03 \pm 1.67$ \\
$\mathrm{IgG}(\mathrm{g} / \mathrm{L})$ & $9.02 \pm 2.14$ \\
$\mathrm{Cr}(\mu \mathrm{mol} / \mathrm{L})$ & $108.6(87.5,172.9)$ \\
$\mathrm{Cys}-\mathrm{C}(\mathrm{mg} / \mathrm{L})$ & $1.89(1.34,2.45)$ \\
eGFR $\left(\mathrm{mL} / \mathrm{min} / 1.73 \mathrm{~m}^{2}\right)$ & $55.94(27.00,75.77)$ \\
\hline
\end{tabular}

DN: diabetic nephropathy, FBG: Fasting blood glucose, Cr: creatinine, Cys-C: cystatin C, eGFR: estimated glomerular filtration. 